\title{
Crianças institucionalizadas e sintomas depressivos por meio do Rorschach e do CDI
}

\author{
Ana Cristina Resende ${ }^{1}$ \\ Lorena de Melo Mendonça Oliveira² \\ Renata Franco ${ }^{3}$ \\ ${ }^{1}$ Pontificia Universidade Católica de Goiás, GO, Brasil \\ https://orcid.org/0000-0001-5730-2577 \\ ${ }^{2}$ Pontifícia Universidade Católica de Goiás, GO, Brasil \\ https://orcid.org/0000-0002-6429-1092 \\ ${ }^{3}$ Institut Catholique de Toulouse, Toulouse, França \\ https://orcid.org/0000-0002-7352-2833
}

\begin{abstract}
Resumo
Os sintomas depressivos em crianças podem ser sutis e variados. Este estudo investigou sintomas depressivos em crianças institucionalizadas para a adoção, comparando-as com crianças não institucionalizadas, utilizando para isso o método de Rorschach Sistema Compreensivo e o CDI. Participaram do estudo 84 crianças, com idades entre 7 e 11 anos. Para a análise de dados foram utilizados: o teste t de student, a correlação de Pearson e o d de Cohen. Os resultados revelaram que as crianças institucionalizadas se mostraram com mais baixa autoestima, problemas afetivos, ideações suicidas, dificuldade escolar e dificuldade na relação com o outro quando comparadas com as crianças não institucionalizadas. Esses dados sugerem uma possível síndrome depressiva, sendo aconselhável a investigação clínica mais aprofundada para providenciar diagnóstico e tratamento, especialmente nas crianças institucionalizadas. Além disso, foram observadas associações entre as variáveis do Rorschach e CDI. Implicações desses achados e sugestões de pesquisas futuras são apresentadas ao final.
\end{abstract}

Palavras-chave: crianças institucionalizadas, depressão infantil, teste de Rorschach, escala de autoavaliação.

\section{Institutionalized children and depressive symptoms by the Rrorschach and CDI}

\begin{abstract}
Depressive symptoms in children can be subtle and varied. This study investigated depressive symptoms in institutionalized children for adoption, comparing them with non-institutionalized children, using the Rorschach method Comprehensive System and CDI. Fifty male and female children took part in this study. These children were between seven and eleven years old. For the analysis of data, it was used: Student's t test, Pearson's correlation, and Cohen's d. The results revealed that the institutionalized children showed lower self-esteem, affective problems, suicidal ideation, school difficulties and impairments in the relationship with the other when compared to non-institutionalized children. These data suggest a possible depressive syndrome, and the further clinical investigation is advisable to provide diagnosis and treatment, especially to institutionalized children. In addition, associations were found between the variables of the Rorschach and CDI. Implications of these findings and suggestions for future research are presented at the end.
\end{abstract}


Keywords: sheltered children, child depression, Rorschach test, self assessment scale.

\title{
Niños institucionalizados y sintomas depresivos a través del Rorschach y CDI
}

\begin{abstract}
Resumen
Los síntomas depresivos en los niños pueden ser sutiles y variados. Este estudio investigó síntomas depresivos en niños institucionalizados para la adopción, comparándolos con niños no institucionalizados, utilizando para ello el método de Rorschach Sistema Comprensivo y el CDI. En el estudio participaron 84 niños, con edades entre 7 y 11 años. Para el análisis de datos se utilizaron: la prueba t de student, la correlación de Pearson y el d de Cohen. Los resultados revelaron que los niños institucionalizados se mostraron con más baja autoestima, problemas afectivos, ideaciones suicidas, dificultad escolar y dificultad en la relación con el otro en comparación con los niños no institucionalizados. Estos datos sugieren un posible síndrome depresivo, siendo aconsejable la investigación clínica más profunda para proporcionar diagnóstico y tratamiento, especialmente en los niños institucionalizados. Además, se observaron asociaciones entre las variables de Rorschach y CDI. Las implicaciones de estos hallazgos y sugerencias de investigaciones futuras se presentan al final.

Palabras clave: niños abrigados, depresión infantil, test de Rorschach, escala de autoevaluación.
\end{abstract}

Os primeiros estudos sobre crianças depressivas aconteceram na Europa, logo após a Segunda Guerra Mundial, quando René Spitz era pediatra de um orfanato. O médico cuidava de crianças que foram retiradas de suas mães por motivo de encarceramento. Spitz (1976) percebeu que os sintomas depressivos se agravavam quando a separação era superior a três meses e que se tornavam irreversíveis quando ultrapassavam cinco meses. Após esse período, apareciam outros transtornos psíquicos que prejudicavam significativamente o desenvolvimento piscoafetivo dessas crianças. O conjunto de sintomas descritos por Spitz passou a ser conhecido como hospitalismo.

As mesmas observações foram reencontradas alguns anos mais tarde na Inglaterra por Bowlby (1978), que descreveu a evolução depressiva de crianças em três etapas: protesto, desespero e desapego. Na primeira etapa a criança chorava e reclamava os pais; na segunda, a criança, desesperada se fechava em si mesma e na terceira etapa, a criança se desapegava dos pais, frequentemente não os reconhecendo mais.

Mais recentemente, baseando-se em pesquisas que estabeleceram os mesmos critérios diagnósticos para as diferentes faixas etárias, a quinta edição do Manual Diagnóstico Estatístico de Transtornos Mentais - DSM 5 (American Psychological Association, [APA] 2014) aponta que o transtorno depressivo maior em crianças, mais comumente, se caracteriza pelo humor irritável ou rabugento, embora a criança também possa apresentar-se chorosa ou com humor deprimido, triste ou abatido, que são mais predominantes nos adultos. A criança ainda pode apresentar diminuição do interesse ou prazer em todas ou quase todas as atividades a maior parte do dia, pode ter insucesso em obter ganho de peso esperado para a idade, como também ter, quase todos os dias, insônia ou hipersonia, agitação ou retardo psicomotor, fadiga ou perda de energia, sentimentos de inutilidade ou culpa excessivas, dificuldade de concentração ou indecisão e, finalmente, pensamentos de morte ou ideações suicidas recorrentes. Para que uma criança seja diagnosticada com depressão maior espera-se que pelo menos cinco desses nove sintomas estejam presentes por um período de duas semanas, e representar uma mudança em relação ao funcionamento anterior, assim como espera-se que pelo menos um dos sintomas seja humor deprimido ou perda de interesse ou prazer.

Outros autores (Calderaro \& Carvalho, 2005; Copper-Royer, 2017) observaram que a Outros autores (Calderaro \& Carvalho, 2005; Copper-Royer, 2017) observaram que a depressão em crianças ainda pode estar associada à sintomas somáticos como cefaleia, dores abdominais, diarreia, enurese, encoprese, além de atrasos psicomotor e de linguagem. Resende e 
Teodoro (2018) também destacam que o diagnóstico da depressão pode ter comorbidade com outros transtornos psicopatológicos na infância, tais como transtorno disruptivo de desregulação do humor (TDDH), que possui a irritabilidade como o fator central (APA, 2014), o transtorno de ansiedade, o transtorno de conduta (TC), o transtorno opositor desafiante (TOD) e o transtorno de de atenção com hiperatividade (TDAH). No TOD o humor irritável é o sintoma que se destaca quando presente juntamente com a depressão. E no TDAH, a dificuldade de concentração em suas atividades que exigem esforço mental constante e a inquietação parecem ser os sintomas centrais e também podem coexistir com os sintomas depressivos na infância. Assim, essa diversidade de sintomas, com diferentes formas de manifestações, limitação da criança em perceber e descrever seus próprios sintomas verbalmente dificulta a realização do diagnóstico da depressão infantil com precisão (Goldman, 2012).

Outro fator que pode dificultar a realizaros transtornos, e a realização de tal diagnóstico em crianças é a falta de instrumentos válidos e padronizados específicos para essa faixa etária. A deficiência de instrumentos para avaliação da personalidade infantil demanda a realização diversificada de estudos que venham promover e expandir a qualidade na prestação de serviços para a população infanto-juvenil (Farah, Cardoso, \& Villemor-Amaral, 2014).

A prevalência de episódios depressivos em crianças tende a variar entre $1 \%$ e $3 \%$ (Resende \& Teodoro, 2018) e isso é considerado uma prevelência alta na área da saúde. Os fatores de risco, aqueles que favorecem a probabilidade de ocorrer de ocorrência dessa doença em crianças, podem ser observados em diferentes âmbitos: no familiar, no individual e no social. Um ambiente familiar hostil, pais ou cuidadores negligentes, abusivos ou agressivos, com problemas emocionais, depressivos ou carentes de afetos e apoio social constituem fatores de risco no âmbito familiar. No âmbito individual, estudos apontam fatores genéticos, o temperamento, a capacidade de regulação emocional e alguns traços de personalidade, tais como, resistência às mudanças, vulnerabilidade cognitiva e pessimismo. No âmbito social, o ambiente violento e a dificuldade de socialização, especialmente na escola, também foram apontados como importantes fatores de risco (Avanci, Assis, Oliveira, \& Pires, 2009; Goldmann, 2012; Resende \& Teodoro, 2018).

Desse modo, o presente estudo investigou a presença de sintomas depressivos em crianças que estavam sob acolhimento institucional para a adoção por falha, omissão ou abuso dos pais. Esse tipo de acolhimento é considerado uma medida de proteção prevista quando os direitos da criança são ameaçados ou violados, como em caso de sofrimento de qualquer forma de negligência, discriminação, exploração, violência, crueldade e opressão (art. 5. ${ }^{\circ}$ e art. 98. ${ }^{\circ}$, incisos I, II e III, ECA). Esse afastamento pode ser a melhor intervenção imediata para a sobrevivência da criança e ou para que ela tenha alguma qualidade de vida, mas as consequências desse afastamento podem ser prejudiciais quando realizado de modo inadequado, sem suporte psicológico e social, ou seja, sem oferecer à criança opções saudáveis para o seu desenvolvimento bio-psico-social (Braithwaite, O’Connor, Degli-Esposti, Luke \& Bowes, 2017).

Oliveira e Resende (2016) observaram associações entre depressão e comportamentos externalizantes em crianças institucionalizadas, diante de diversas situações adversas sofridas por elas, a partir de quatro meses de institucionalização. Considerando um período mínimo de dois anos de acolhimentos, Alvarez e Lobato (2013) notaram um maior índice de sintomas depressivos em crianças que não tinham irmãos na instituição, que tiveram um acolhimento sem padrinhos e que foram acolhidas depois dos sete anos de idade. E o estudo de Batki (2017) demonstrou que a institucionalização de crianças estava relacionada com menor capacidade para a regulação emocional, ou seja, menos habilidade para perceber, administrar e expressar as emoções para atingir metas estabelecidas ou para adaptar psicologicamente dentro de um contexto social. A falta dessa regulação pode gerar vários sintomas emocionais e 
comportamentais, que a longo prazo, podem prejudicar o desenvolvimento de crianças adotadas.

Outros estudos (Calcing \& Benetti, 2014; Pracana \& Santos, 2010; Rutter, Kumsta, Schlotz, \& SonugaBarke, 2012) têm demonstrado que a institucionalização poderá ter um impacto negativo em diferentes áreas do desenvolvimento da criança devido às inadequações na forma como se estabeleceram as relações e o contato afetivo com os progenitores e as circunstâncias de perda ou separação. Normalmente, as relações dessas crianças com os cuidadores eram de maus tratos, negligência, abandono e as circunstâncias eram de carência parental. Todos esses aspectos podem ser centrais no processo de desenvolvimento da patologia depressiva ou de estresse pós-traumático ou de dificuldades crônicas no estabelecimento de vínculos interpessoais saudáveis (Braithwaite et al., 2017; Sperry \& Widom, 2013).

Esses estudos também relataram dificuldades significativas nas crianças em situação de acolhimento para acompanharem o nível de escolaridade adequado para a idade, ou para completar o ensino médio. Outros aspectos observados nessas crianças foram: o envolvimento mais frequente com transgressões de regras sociais e com o consumo abusivo de substâncias psicotrópicas, bem como a predisposição para serem vitimizados e revitimizados fisicamente ou psicologicamente, a tendência para a paternidade precoce, e ainda ter seus próprios filhos institucionalizados para adoção (Bakti, 2017; Wulczyn, Brunner, \& Goerge, 2002).

Segundo Gouveia e Gouveia (2013), existem diferentes estratégias para conhecer se a criança apresenta depressão: entrevistá-las diretamente, obter informações de seus pais ou responsáveis e de pessoas que possam ter um papel de destaque em sua vida como avós, tios e/ ou professores, observar o seu comportamento durante atividades diversas como em situação de contato social, quando é requerida a demonstração de afetos e/ou em contextos solitários, além de empregar questionários, inventários de autorrelatos e testes projetivos.
Sendo assim, este estudo associou o Inventário de Depressão Infantil - CDI, um instrumento de autorrelato, com o Método de Rorschach de acordo com o Sistema Compreensivo (SC), um instrumento projetivo. O instrumento de autorrelato permite à criança expressar, de acordo com o seu próprio julgamento, como ela pensa, sente e se comporta diante de uma situação geralmente simples e familiar. Contudo, essas informações são limitadas ao que a criança é capaz de dizer sobre si. Isso depende da sua capacidade cognitiva, da sua motivação para responder e do quão consciente elas estão das suas características e comportamentos (Meyer \& Kurtz, 2006).

O método de Rorschach, por sua vez, se fundamenta na observação de como a criança executa a tarefa que é definida por ela mesma, com poucos limites de como deve ser feita. Uma vantagem desse instrumento é que, devido à metodologia indireta que emprega, ele é mais propício para revelar características de personalidade que a criança não reconhece plenamente em si ou hesita em admitir quando questionada sobre elas diretamente. Ou seja, o instrumento projetivo pode complementar as informações levantadas pelos instrumentos de autorrelato (Weiner \& Greene, 2008). Salienta-se que apesar das vantagens, não foram identificados, nos últimos dez anos, estudos com amostras de crianças institucionalizadas para adoção com esse instrumento. Assim, acredita-se que os dois tipos de instrumentos (CDI e Rorschach SC) com propósitos de avaliação diferenciados permite dar um panorama geral do funcionamento psíquico das crianças institucionalizadas no que diz respeito aos sintomas depressivos, de forma que o resultado de um instrumento complementa o resultado do outro.

Diante do que foi descrito, o presente estudo teve como objetivo geral revelar uma perspectiva do funcionamento psíquico das crianças institucionalizadas no que diz respeito aos aspectos afetivos, mais especificamente sobre os sintomas depressivos. Para tanto, avaliou-se os sintomas depressivos e comportamentais em crianças em situação de acolhimento institucional 
comparando-as com crianças que viviam com suas famílias, provenientes do mesmo contexto socioeconômico, por meio do Rorschach SC e do CDI.

\section{Metodologia}

\section{Participantes}

Participaram deste estudo, após a exclusão de três participantes, 84 crianças, entre sete e onze anos de idade, todas voluntárias e autorizadas por um de seus responsáveis por meio do Termo de Consentimento Livre e Esclarecido - TCLE. As crianças foram divididas em dois grupos: Grupo 1 (G1), constituído por crianças institucionalizadas para a adoção, e Grupo 2 (G2) composto por crianças não institucionalizadas. No G1 havia 42 crianças selecionadas em três instituições de acolhimento indicadas pelo Juizado da Infância e Juventude da cidade, sendo 26 do sexo masculino e 16 do sexo feminino, encaminhadas a essas instituições por diferentes motivos que envolviam risco à vida: violência sexual, psicológica, física ou negligência. $\mathrm{O}$ critério de inclusão para o G1 foi ter a idade entre 7 e 11 anos. Os critérios de exclusão para esse mesmo grupo foram a ausência de informações sobre as circunstâncias que levaram à institucionalização e a indisposição da criança para realizar as atividades de pesquisa que foram propostas. Para cada um desses critérios, uma criança foi excluída. A primeira instituição abrigava somente crianças do sexo masculino. Todas aquelas que atenderam ao critério de inclusão participaram do estudo ( $\mathrm{n}=$ $16,100 \%$ ). Nas demais instituições, foram selecionadas 13 crianças em cada uma delas, considerando o critério de conveniência e os critérios de inclusão e exclusão.

O G2 foi composto por outras 42 crianças de três escolas municipais, provenientes das mesmas regiões das crianças do G1. Essas crianças foram pareadas em termos de sexo, idade e nível socioeconômico com as crianças do G1. Entende-se que, assim, pode-se minimizar possíveis variáveis de confundimento, tornando a variável acolhimento institucional um dos principais aspectos discrepante entre os dois grupos. O critério de exclusão adotado foi ter passado por qualquer situação prévia de acolhimento institucional e estar sob tratamento medicamentoso, psiquiátrico e/ou psicológico, considerando as informações fornecidas pelos pais ou responsáveis. Nesse grupo, somente uma criança foi excluída por estar sob tratamento psicológico. Ao total foram contatados 60 pais ou responsáveis pelas crianças (20 em cada escola), mas somente $70 \%(\mathrm{~N}=42)$ deles tiveram a disponibilidade de participar da pesquisa no momento da coleta de dados que era realizada nas escolas.

\section{Instrumentos}

Protocolo para registro de dados coletados nos prontuários das crianças do G1. Auxiliou na coleta de informações significativas sobre a história de vida, motivo do acolhimento, tempo de acolhimento e nível socioeconômico dessas crianças.

\section{Questionário Sociodemográfico para as crian-} ças do G2 (não institucionalizadas). Foi respondido pelos responsáveis e utilizado para coletar dados que descreviam os participantes do estudo com informações gerais tais como: sexo, idade, local de residência, renda familiar, estrutura familiar, desempenho escolar, comportamento, tratamentos realizados e se a criança já esteve em situação de acolhimento institucional.

Inventário de Depressão Infantil (Children's Depressory Inventory - CDI). Criado por Kovacs em 1983, nos Estados Unidos, a partir de uma adaptação do Beck Depression Inventory para adultos, para abordar o constructo da depressão em criança de 7 a 17 anos. O instrumento é composto por 27 itens, com três alternativas de resposta sob forma de afirmativas, dentre as quais a criança deve escolher a que melhor descreve o seu estado no período atual. Cada afirmativa tem uma pontuação que varia de 0 a 2 indicando a severidade dos sintomas na ordem crescente de pontuação. Os critérios de correção dos dados coletados foram baseados nas normas estabelecidas no estudo de Wathier et al. (2008), o qual 
demonstrou boa consistência interna do instrumento (alfa de Cronbach de 0,85). O escore total é calculado pela soma das pontuações referentes às escolhas da criança, que é transformada em um percentil estimado, considerando o sexo e a idade da criança. Classifica-se clinicamente significativo o percentil a partir de 85 .

\section{Método de Rorschach (Sistema Compreensivo}

- SC). Esse instrumento foi elaborado por Hermann Rorschach em 1921, na Suíça. Trata-se de uma técnica projetiva e psicométrica, construída para avaliar uma ampla gama de características de personalidade. É composta por dez cartões, cinco contendo manchas escuras e outros cinco contendo manchas coloridas, os quais sevem de estímulos pouco organizados que levam o indivíduo em avaliação a expressar conteúdos associativo-perceptivos representativos de seu mundo interno. A aplicação é individual e exige que os examinandos identifiquem o que os borrões de tinta construídos parecem em resposta à pergunta "O que isso poderia ser?". Cada resposta ou solução para a tarefa é codificada de acordo com as orientações padronizadas através de um número de dimensões e os códigos são, então, resumidos em escores e, posteriormente, interpretados seguindo o Sistema Compreensivo (Exner, 2003). As normas utilizadas para o teste são específicas para a população brasileira, publicadas por Nascimento, Resende e Ribeiro (2017). Para este estudo, foram avaliadas as variáveis referentes ao Índice de Depressão - DEPI (valor total), aos Conteúdos Críticos (Soma de An, Bl, Ex, Fi, Fd, Sx, Xy, AG e MOR) que apontam para experiências traumáticas, e aos aspectos afetivos. As variáveis do aspecto afetivo foram: WsumC (capacidade de expressar os afetos); FC+CF (modulação dos afetos e espontaneidade afetiva); C (violentas descargas afetivas); Sum C' (índice de constrição afetiva) C', Y, T, V (experiências afetivas dolorosas e desagradáveis); SumV (sentimento de culpa e ressentimentos); Sum Sombreado (estresse emocional); CP (falsas emoções positivas típica de mecanismos bipolares/ciclotímicos); Afr (índice de afetividade); $2 \mathrm{AB}+\mathrm{Art}+\mathrm{Ay}$ (índice de intelectualização); $\mathrm{S}$ (raiva e res- sentimentos); Blends (manejo de estimulações afetivas mais complexas); Cor-Sombreado Blends (ambivalência afetiva); $3 r+(2) / R$ (índice de Egocentrismo/ autoesti$\mathrm{ma}$ ); $2 \mathrm{Na}+\mathrm{Ls}+\mathrm{Bt}+\mathrm{Ge}+2 \mathrm{Cl} / \mathrm{R}$ (índice de isolamento); FD (capacidade de se perceber em relação aos outros); MOR (autocríticas negativas); e COP (predisposição para comportamentos cooperativos).

\section{Procedimentos}

Esse estudo caracterizou-se por ser de risco mínimo aos participantes e foi aprovado pelo Comitê de Ética da Pontifícia Universidade Católica de Goiás, tendo seguido os preceitos éticos que regem a realização de pesquisas com seres humanos, conforme Resolução 0466/12 do Conselho Nacional de Saúde e Resolução do Conselho Federal de Psicologia 016/2000. O projeto de pesquisa foi apresentado ao Juiz responsável pelo Juizado da Infância e Juventude de Goiânia, o qual, após ficar ciente da natureza do estudo e aprovar a execução do mesmo, autorizou o acesso às instituições de acolhimento.

Concomitante a esse processo, o projeto de pesquisa também foi apresentado à Secretaria Municipal de Educação de Goiânia. Após aprovação da realização da pesquisa, o departamento responsável por pesquisas daquela secretaria indicou as escolas municipais nas regiões de interesse das pesquisadoras. Toda a coleta de dados foi realizada pelas duas primeiras autoras desse artigo, que tinham experiência em atendimento psicológico com crianças e docência universitária.

No que tange ao G1, após a autorização do responsável pela criança, a pesquisadora utilizou o Protocolo para registro de dados coletados nos prontuários das crianças. No que diz respeito ao G2, os seus responsáveis foram contatados por telefone, informados brevemente sobre a natureza da pesquisa e, caso se interessavam pela participação da criança no estudo, era agendado um horário na própria escola para maiores esclarecimentos,, assinatura do TCLE, e preenchimento do questionário sociodemográfico.

Os outros instrumentos (CDI e Rorschach SC) foram aplicados pelas pesquisadoras nas próprias instituições 
de acolhimento e de ensino, de forma individual e em horários combinados com antecedência respeitando a rotina de cada criança. Nenhuma criança apresentou resistência durante a execução das tarefas propostas. O tempo médio de aplicação do CDI foi de 20 minutos e do Rorschach SC 40 minutos. Considerando o tempo para explicar o objetivo do estudo e obter o assentimento de cada criança, o tempo total de coleta de dados com cada um delas foi de aproximadamente 70 minutos, divididos em dois encontros, sendo o primeiro para obter o assentimento da criança e a aplicação do CDI e o segundo para a aplicação do Rorschach.

Os protocolos foram codificados de acordo com as proposições técnicas de cada instrumento. No caso do Rorschach, por ser um teste mais complexo para ser corrigido, todos os protocolos foram codificados pela primeira autora deste trabalho e, para verificar a confiabilidade da codificação dos testes, $25 \%$ de todos os protocolos úteis $(\mathrm{N}=21$, respostas $=438)$, selecionados aleatoriamente, também foram codificados de forma independente por outra psicóloga, qualificada para codificação do instrumento, que desconhecia os objetivos deste estudo, para que o exame de concordância entre dois juízes (primeira autora e a psicóloga convidada), por meio do coeficiente Kappa, fosse realizado com a finalidade de garantir a qualidade da codificação das respostas como condição necessária para análise dos dados. Os resultados foram considerados satisfatórios (Kappa $>0,70)$ e indicaram que a correção do método de Rorschach para esse estudo foi considerada confiável. Os percentuais de concordância variaram de 0,82 (Conteúdos com 27 categorias) a 1,00 (categoria de Localização e Espaço com duas variáveis) e os valores Kappa foram: 0,80 (Escore Z); 0,82 (Conteúdos, 27 variáveis), 0,80 (Códigos Especiais com 14 variáveis), 0,86 (Pares), 0,88 (Determinantes com 11 variáveis); 0,90 (FQ), 0,91 (DQ), 0,97 (Localização e Espaço com quatro variáveis) e 0,99 (categoria de Localização e Espaço com duas variáveis).
Os dados foram analisados por meio do programa estatístico SPSS - Statistical Package for the Social Sciences - versão 18.0. Inicialmente, aplicou-se o teste de normalidade Kolmogorov-Smirnov que constatou que todas as variáveis do estudo apresentavam uma distribuição normal. A partir dessa análise, os resultados foram descritos mediante estatística descritiva (média e desvio padrão), correlacional (Correlação de Pearson) e comparativa (Teste t de Student para amostras independentes) para os dados do CDI e para todas as variáveis selecionadas do Rorschach. O nível de significância adotado foi de 0,05 . Além desses índices foram incluídos os d de Cohen que avaliam o tamanho do efeito das diferenças ente os grupos, sendo considerado um tamanho do efeito grande maior do que 0,8 (Cohen, 1988).

\section{Resultados e discussão}

No que se refere às crianças do G1 (institucionalizadas para a adoção), após analisar os seus prontuários, verificou-se que eram originárias de várias regiões socioeconômicas desfavorecidas do Estado. Sessenta por cento dessas crianças eram provenientes de duas regiões de risco que esbarravam na territorialização do tráfico de drogas e violência, onde o poder público praticamente não lançava projetos de inclusão dessa população pobre, de maioria analfabeta e sem qualificação profissional (Peixoto et al., 2012). O acesso à saúde, educação, transporte e saneamento eram bem precários nessa região.

Os motivos dos acolhimentos descritos nos prontuários foram: risco social, abandono de incapaz, abuso sexual, maus tratos, violência doméstica, violência física e medida protetiva. Em alguns prontuários constavam mais de um desses motivos. Quanto ao tempo de acolhimento, variou de um mínimo de quatro meses a um máximo 35 meses $(M=13,56, \mathrm{DP}=8,86)$. Todas as crianças do G1 eram de famílias de classe socioeconômica baixa e pelo menos um de seus genitores tinha envolvimento com drogas. A idade média dos participantes foi de 9,38 anos $(\mathrm{DP}=1,48)$. Todos estavam 
frequentando a escola regularmente e a média de anos de estudo por criança foi de 2,6 $(\mathrm{DP}=1,36)$. A maior parte das crianças $(62 \%, \mathrm{n}=26)$ era do sexo masculino.

No que tange ao G2 (grupo de crianças não institucionalizadas), sua constituição foi pareada ao G1 em termos de quantidade de participantes, sexo, idade e aspectos socioeconômico. Em relação aos anos de estudo, esse grupo apresentou a média de 3,1 anos (DP= 1,07), revelando diferença estatisticamente significante entre $\mathrm{G} 1$ e $\mathrm{G} 2$, com o tamanho do efeito considerado pequeno $(t=2,39, p=0,05$ e $d=0,41)$. Esse achado em relação à escolaridade corrobora um aspecto comumente observado em crianças que foram institucionalizadas para adoção por negligência e por ter sofrido algum tipo de vitimização. Vários estudos sustentam que existe conexão entre o desempenho escolar e o aspecto afetivo, em que crianças vitimizadas frequentemente apresentam dificuldade de aprendizagem, baixo rendimento escolar e até mesmo prejuízos em funções neurocognitivas como a inteligência (Braithwaite et al., 2017; Dell'Aglio \& Hutz, 2004; Milani \& Loureiro, 2009; Paredes \& Calvete, 2014; Pereira, Santos, \& Williams, 2009). Essa questão do desempenho escolar prejudicado em crianças institucionalizadas é muito importante. Isso pode interferir em todo o seu desenvolvimento posterior, especialmente no seu desenvolvimento cognitivo, o que acaba sendo uma situação de vulnerabilidade para ela sofrer bulling na escola, ser isolada socialmente, ou ficar a mercê de novas vitimizações. Por isso a intervenção com essas crianças é preventiva não do abandono, mas preventiva de toda uma história de complicações posteriores (Gontijo \& Medeiros, 2007).

\section{Resultados do CDI}

Ao comparar os resultados dos grupos de uma forma geral, ou separando as crianças por sexo, obteve-se os seguintes resultados observados na Tabela 1.

\section{Tabela 1.}

Média, desvio padrão, valor do teste t e d de Cohen em relação aos resultados do CDI, por sexo e por grupo

\begin{tabular}{|c|c|c|c|c|c|c|c|c|c|c|c|c|c|c|c|c|}
\hline \multirow{2}{*}{ Contexto } & \multirow{2}{*}{$\mathbf{N}$} & \multicolumn{5}{|c|}{ Feminino $(n=16)$} & \multicolumn{5}{|c|}{ Masculino $(n=26)$} & \multicolumn{5}{|c|}{ Total $(N=42)$} \\
\hline & & $M$ & $D P$ & $t$ & Sig & $d$ & $M$ & $D P$ & $t$ & Sig & $D$ & $M$ & $D P$ & $t$ & Sig & $d$ \\
\hline G1 & 42 & 13,9 & 5,3 & \multirow{2}{*}{4,0} & \multirow{2}{*}{$*$} & \multirow{2}{*}{2,32} & 13,3 & 6,5 & \multirow{2}{*}{2,9} & \multirow{2}{*}{$0,04^{*}$} & \multirow{2}{*}{0,96} & 13,6 & 5,1 & \multirow{2}{*}{3,9} & \multirow{2}{*}{ * } & \multirow{2}{*}{1,7} \\
\hline $\mathrm{G}_{2}$ & 42 & 5,2 & 2,2 & & & & 8,2 & 4,1 & & & & 6,7 & 2,6 & & & \\
\hline
\end{tabular}

$M=$ média; $D P=$ desvio padrão. ${ }^{*} \mathrm{p}=0,01$ para o teste $t$ de Student

Comparando os resultados do escore bruto do CDI entre os grupos (G1 e G2), encontrou-se diferenças estatisticamente significantes e tamanhos de efeito grande. Esses resultados se mantiveram quando foram considerandas as diferenças entre os sexos nas comparações intergrupos (Tabela 1). Esses dados apontam que aqueles participantes provenientes de instituições de acolhimento para a adoção tendem a apresentar médias maiores de sintomas depressivos. No G1, 35,7\% ( $\mathrm{n}=15)$ dos participantes foram considerados clinicamente sig- nificativos em relação à sintomatologia depressiva, e no G2 aproximadamente $10 \%(n=4)$. Considerando o estudo de correlação, houve uma associação significativa entre a sintomatologia depressiva e crianças institucionalizadas $(\mathrm{r}=0,38 ; p=0,02)$, indicando que essas crianças tendem a ter mais sintomas depressivos do que as crianças não institucionalizadas que vivem com suas famílias.

Considerando os grupos separadamente (G1 e G2) não foram encontradas diferenças significativas intragrupos na pontuação entre meninos e meninas. Essa diferença 
entre sexos, dentro de um mesmo grupo, também não foi evidenciada no estudo de Oliveira e Resende (2016).

Para verificar se os sintomas depressivos se manifestavam de forma diferente entre as crianças do G1 e do G2, realizou-se uma análise dos itens do CDI. Os itens que apresentaram diferença significativa estão listados na Tabela 2.

\section{Tabela 2.}

Média, desvio padrão, valor do teste $\mathrm{t}$ e $\mathrm{d}$ de Cohen dos itens do CDI que apresentaram diferença significativa entre os grupos de crianças.

\begin{tabular}{|c|c|c|c|c|c|c|}
\hline Itens do CDI & Grupo & $\mathbf{M}$ & DP & $t$ & Sig. & d \\
\hline \multirow{2}{*}{ 7. Eu me detesto. } & G1 & 0,73 & 0,30 & \multirow{2}{*}{2,472} & \multirow{2}{*}{$0,02^{*}$} & \multirow{2}{*}{2,6} \\
\hline & G2 & 0,07 & 0,21 & & & \\
\hline \multirow{2}{*}{ 9. Eu quero me matar } & G1 & 0,48 & 0,30 & \multirow{2}{*}{3,12} & \multirow{2}{*}{$0,00^{*}$} & \multirow{2}{*}{3,2} \\
\hline & G2 & 0,00 & 0,00 & & & \\
\hline \multirow{2}{*}{ 10. Tenho vontade de chorar todos os dias. } & G1 & 0,77 & 0,44 & \multirow{2}{*}{3,57} & \multirow{2}{*}{$0,00^{*}$} & \multirow{2}{*}{2,3} \\
\hline & G2 & 0,07 & 0,16 & & & \\
\hline \multirow{2}{*}{ 15. Eu tenho sempre que me forçar para fazer minhas lições escolares. } & G1 & 1,54 & 0,52 & \multirow{2}{*}{4,04} & \multirow{2}{*}{$0,00^{*}$} & \multirow{2}{*}{2,5} \\
\hline & G2 & 0,20 & 0,54 & & & \\
\hline \multirow{2}{*}{ 23. Estou indo mal em matérias que antes eu ia bem. } & G1 & 1,00 & 0,43 & \multirow{2}{*}{4,52} & \multirow{2}{*}{$0,00^{*}$} & \multirow{2}{*}{2,4} \\
\hline & G2 & 0,11 & 0,32 & & & \\
\hline \multirow{2}{*}{ 24. Nunca vou ser tão bom quanto os outros } & G1 & 0,83 & 0,33 & \multirow{2}{*}{4,12} & \multirow{2}{*}{$0,00^{*}$} & \multirow{2}{*}{2,2} \\
\hline & G2 & 0,11 & 0,32 & & & \\
\hline \multirow{2}{*}{ 27. Eu me meto em brigas o tempo todo. } & G1 & 0,79 & 0,51 & \multirow{2}{*}{2,15} & \multirow{2}{*}{$0,03^{*}$} & \multirow{2}{*}{1,9} \\
\hline & G2 & 0,11 & 0,22 & & & \\
\hline
\end{tabular}

$M=$ média; $D P=$ desvio padrão. ${ }^{*} \mathrm{p}<0,05$ para o teste $t$ de Sutdent

Todos os itens de teste citados na Tabela 2 tiveram escores maiores no G1 e com tamanho do efeito considerado grande para as comparações entre os dois grupos $(\mathrm{d} \geq 1,9)$. Ou seja, as crianças institucionalizadas (G1) apresentaram mais baixa autoestima (itens 7 e 24), assim como mais problemas afetivo-somáticos, incluindo aqui ideações suicidas (itens 9 e 10), mais dificuldade escolar (item 15 e 23) e dificuldade na relação com o outro (item 27) quando comparadas com as crianças não institucionalizadas que vivem com suas famílias (G2).
De acordo com a literatura, esses sintomas mais frequentes no G1 são comuns em crianças com depressão (APA, 2014). Esses achados também confirmam os dados da literatura que sustentam que crianças que vivem em instituições de acolhimento apresentam mais sintomas depressivos do que as crianças que vivem com suas famílias (Alvarez \& Lobato, 2013; Oliveria \& Resende, 2016; Wathier et al., 2008). Esse dado aponta que a institucionalização por motivos de sérios problemas familiares antecedentes, tais como negligêna e 
agressividade por parte dos pais, pode ter um impacto negativo em diversas áreas do desenvolvimento da criança, especialmente no que diz respeito à afetividade, autopercepção, relacionamentos interpessoais (Bick \& Nelson, 2016).

\section{Resultados do Rorschach SC}

A Tabela 3 mostra as análises estatísticas descritivas e comparativas entre os grupos das cinco variáveis relacionadas aos aspectos afetivos, que apresentaram diferença significativa entre os grupos de crianças e tamanhos de efeito grandes.

\section{Tabela 3.}

Média, desvio Padrão, valor do teste t e do d de Cohen em relação às variáveis dos aspectos afetivos do Rorschach SC.

\begin{tabular}{|c|c|c|c|c|c|c|}
\hline Variáveis do Rorschach & Grupo & M & DP & $T$ & Sig. & d \\
\hline $\mathrm{FC}+\mathrm{CF}$ & $\begin{array}{l}\text { G1 } \\
\text { G2 }\end{array}$ & $\begin{array}{l}1,50 \\
2,53\end{array}$ & $\begin{array}{l}0,50 \\
1,04\end{array}$ & $-2,53$ & $<0,01^{*}$ & $-1,34$ \\
\hline $\mathrm{C}$ & $\begin{array}{l}\text { G1 } \\
\text { G2 }\end{array}$ & $\begin{array}{l}0,55 \\
0,04\end{array}$ & $\begin{array}{l}0,67 \\
0,15\end{array}$ & 2,21 & $0,04^{*}$ & 1,24 \\
\hline Índice Egocentrismo & $\begin{array}{l}\text { G1 } \\
\text { G2 }\end{array}$ & $\begin{array}{l}0,15 \\
0,31\end{array}$ & $\begin{array}{l}0,11 \\
0,15\end{array}$ & $-2,78$ & $0,02^{*}$ & $-1,21$ \\
\hline $\mathrm{COP}$ & $\begin{array}{l}\text { G1 } \\
\text { G2 }\end{array}$ & $\begin{array}{l}0,26 \\
1,07\end{array}$ & $\begin{array}{l}0,62 \\
1,07\end{array}$ & $-2,40$ & $0,02^{*}$ & $-0,93$ \\
\hline Conteúdos Críticos & $\begin{array}{l}\text { G1 } \\
\text { G2 }\end{array}$ & $\begin{array}{l}3,83 \\
2,45\end{array}$ & $\begin{array}{l}1,08 \\
1,64\end{array}$ & 2,99 & $0,04^{*}$ & 0,99 \\
\hline
\end{tabular}

$M=$ média; $D P=$ desvio padrão. $* \mathrm{p}<0,05$ para o teste $t$ de Sutdent

* A contribuição individual de cada autora: a primeira autora trabalhou na concepção do projeto de pesquisa, na metodologia, coleta de dados e na redação do artigo; a segunda trabalhou na concepção do projeto de pesquisa, na coleta de dados e redação do artigo; terceira autora trabalhou na redação do artigo.

Considerando os resultados da variável $\mathrm{C}(p=$ $0,02)$, notou-se que as crianças do G1 tendem mais frequentemente a expressar seus afetos sem modulação, mediante violentas descargas afetivas, mostrando-se frequentemente mais imaturas e intempestivas do que as crianças da mesma idade do G2. Assim, crianças do G1 apresentaram mais dificuldade em controlar cognitivamente ou modular as suas emoções, apresentando mais labilidade, instabilidade afetiva e reações afetivas mais agressivas e inapropriadas para a idade delas (Exner, 2003; Nascimento et al., 2017). As crianças do $\mathrm{G} 2$ revelaram mais espontaneidade, calor nas trocas afetivas, capacidade de modular os afetos e, até mesmo, foram mais egocêntricas em relação aos seus sentimentos, sem, contudo, mostrarem-se desajustadas $(\mathrm{FC}+\mathrm{CF})$. Esse achado corrobora os achado de Batki (2017), que observou maior capacidade de regulação emocional em crianças que viviam com suas famílias de origem quando comparadas com as crianças que foram adotadas quando ainda recém-nascidas ou adotadas tardiamente. As crianças adotadas sempre estavam em desvantagens quando tinham que lidar com situações emocionalmente intensas ou estressantes.

A variável Índice de Egocentrismo $(\mathrm{p}=0,02)$, significativamente menor no G1 $(\mathrm{M}=0,15)$, aponta que essas crianças revelaram uma menor autoestima, menos autoconfiança e tendência a se compararem de modo desfavorável em relação às demais crianças. Este índice 
no G1 também foi menor que aquele encontrado em crianças com desenvolvimento padrão (Nascimento et al., 2017), o que indica que as crianças do G1 também estão mais suscetíveis a serem manipuladas por outras pessoas, uma vez que não acreditam em seus próprios recursos e capacidades, bem como podem estar mais suscetíveis à angústica, tristeza e depressão.

Em relação à variável COP $(p=0,02)$, os dados sugerem que as crianças do G2 $(\mathrm{COP}=1,07)$ tendem a ser mais acolhedoras, cooperativas e simpáticas nas relações interpessoais (Exner, 2003; Resende, 2016) do que as crianças do G1 (COP=0,26). Nesse sentido, o G1 também alcançou desempenhos menores do que a amostra normativa de crianças no estudo de Nascimento et al. (2017), o que corrobora com a análise de que as crianças do G1 revelaram menos habilidades sociais para antecipar atitudes solícitas em seus relacionamentos e tendem a acreditar menos que as interações com os outros serão amigáveis ou de cooperação. Os resultados dessas duas últimas variáveis (Índice de Egocentrismo e COP) permitem inferir que as crianças institucionalizadas para a adoção por terem sido negligenciadas, vítimas de violência doméstica, física e ou sexual foram impactadas de forma em seu desenvolvimento psicológico, especialmente na autopercepção e relacionamentos interpessoais, que a médio ou longo prazo podem desencadear sintomas depressivos (Resende, 2016).

Por meio dos resultados obtidos pela variável Conteúdos Críticos nos dois grupos, observou-se que as crianças do G1 também manifestaram muito mais indícios de sintomas de estresse pós-traumático do que as crianças do G2. A frequência elevada dessa variável tem sido constatada em pessoas vítimas de abuso sexual, espancamento ou outros tipos de violência (Kaser-Boyd, \& Evans, 2008; Luxenberg \& Levin, 2004). Além disso, Batki (2017) também observou significativamente mais conteúdo agressivo ou destrutivo nas avaliações psicológicas de crianças adotadas do que em crianças que sempre foram criadas por sua família de origem. De acordo com a autora, esses conteúdos agressivos e destrutivos poderiam implicar em vários aspectos do mundo interior das crianças adotadas, incluindo suas fantasias, conflitos, dificuldades ou medos, bem como poderiam refletir suas experiências reais e anteriores, como a revivência de experiências traumáticas.

Finalmente, investigou-se como as variáveis do CDI e do Rorschach se integravam para a compreensão dos aspectos afetivos das crianças. Para levantar esta informação, realizou-se, por meio da correlação de Pearson, a correspondência entre os itens do CDI e as seguintes variáveis do Rorschach: $\mathrm{FC}+\mathrm{CF}, \mathrm{C}$, Índice de Egocentrismo, COP, e Conteúdos Críticos do Rorschach.

Os resultados mostraram correlações negativas significantes, porém fracas, entre a variável Índice de Egocentrismo do Rorschach e os itens $7(\mathrm{r}=-0,30, \mathrm{p}=$ $0,04)$ e $24(\mathrm{r}=-0,31, \mathrm{p}=0,03)$ do CDI. Isso aponta uma relação entre baixa autoestima no Rorschach com itens que também apontam autoestima prejudicada no CDI por meio das verbalizações "Eu me detesto" e "Nunca vou ser tão bom quanto os outros". Esse resultado reforça a validade dos instrumentos para captar prejuízos na autopercepção que interferem no bem-estar do indivíduo, como também poderia ser um indício de validade convergente entre essas variáveis (Ego e itens 7 e 24). Outra correlação positiva fraca, porém significativa, foi entre o item 10 do CDI, "Tenho vontade de chorar todos os dias", e a variável Conteúdos Críticos do Rorschach $(r=0,29, p=0,03)$. Essa correlação aponta uma relação direta entre choro e relembrar situações traumáticas.

\section{Consideraçõe finais}

Destaca-se que foi avaliada a presença de mais sintomas depressivos no grupo de crianças institucionalizadas para a adoção do que no grupo de crianças pareadas não institucionalizadas, mas não no sentido de diagnóstico nosológico, tendo em vista a utilização dos instrumentos CDI e Método de Rorschach SC, que permitem o rastreamento da sintomatologia e não a sua confirmação diagnóstica. A presença desses 
sintomas estatisticamente mais frequentes no grupo das crianças institucionalizadas, e em um grau acima da média da amostra normativa, sugere uma possível síndrome depressiva, sendo aconselhável a investigação clínica mais aprofundada para providenciar diagnóstico e tratamento (Alvarez \& Lobato, 2013; Oliveira \& Resende, 2016; Wathier et al., 2008). Assim, as crianças institucionalizadas mostraram-se com mais baixa autoestima, problemas afetivo-somáticos, ideações suicidas, dificuldade escolar e dificuldade na relação com o outro quando comparadas com as crianças não institucionalizadas que vivem com suas famílias.

Um resultado inesperado foi o índice de depressão (DEPI) no Rorschach ser semelhante entre os dois grupos de crianças (institucionalizadas para a adoção e aquelas que viviam com suas famílias de origem), ou seja, não houve diferenças significativas entre o G1 $(\mathrm{DEPI}=4,5)$ e G2 (DEPI= 3,8), mas ambos com médias superiores ao esperado quando comparadas com a média das crianças com desenvolvimento típico do estudo de Nascimento et al. (2017). Esses dados permitem levantar duas hipóteses. A primeira é que as crianças institucionalizadas ainda não apresentam de fato um transtorno grave de depressão. A justificativa é que, se as crianças institucionalizadas, embora estejam demonstrando comportamentos mais típicos de crianças com sintomas depressivos em situações simples e cotidianas, em que elas geralmente sabem o que é esperado delas, mas não demonstram isso por meio do Rorschach (que investiga as reações em situações mais complexas e pouco estruturadas, em que elas não sabem o que é esperado delas), muito provavelmente, as crianças institucionalizadas ainda não apresentam esse transtorno. Ou seja, os sintomas de depressão pelo CDI podem se configurar como um pedido de ajuda de alguém que está para submergir, mas ainda tem forças para gritar, para reagir de modo que as pessoas percebam que ela não sabe o que fazer. Isso também significa que, se a criança for submetida a tratamento, esses problemas podem ser remediados em curto período de tempo.
A segunda hipótese, e a mais provável, referente às médias do G1 e G2 serem maiores do que a média de um grupo de referência local e também a um grupo de referência nacional para o índice de depressão (DEPI) no Rorschach, consiste em se perguntar se o próprio ambiente, de onde essas crianças são provenientes, não as predispõem a uma tendência semelhante para vivenciarem mais períodos de tristeza do que as crianças de uma forma geral, provenientes de contextos menos vulneráveis. É importante ressaltar que todas as crianças desse estudo eram provenientes de áreas de risco que esbarravam na territorialização do tráfico de drogas e da violência, em que o poder público praticamente não lançava projetos de inclusão dessa população pobre, de maioria analfabeta e sem qualificação profissional (Peixoto et al., 2012). Ou seja, da mesma forma que o ambiente doméstico negligente e violento (no caso do G1) pode se tornar propício ao desenvolvimento de sintomas depressivos, a comunidade violenta também pode gerar essa mesma predisposição nas crianças do G2, destacando a relevância da influência do suporte social e comunitário para o desenvolvimento infantil. Até mesmo a exposição a uma vizinhança violenta, ou a exposição indireta à violência, como ser próximo de uma pessoa que seja autora ou vítima de atos violentos pode ser um dos principais componentes para o surgimento de angústias, tristezas e problemas de comportamentos (Avanci et al., 2009).

Ao final da avaliação, percebeu-se que o índice de depressão (DEPI) do Rorschach SC foi sensível para avaliar sintomas depressivos em crianças em situações de risco, como eram as crianças do G1 e G2, mas não foi sensível o suficiente para discriminar diferentes predisposições para a depressão em crianças institucionalizadas e não institucionalizadas provenientes de níveis socioeconômicos desfavorecidos. É importante considerar também que a depressão abrange sintomas somáticos, tais como cefaleia, dores abdominais, alterações no sono (insônia ou hipersonia), no apetite e no peso, que não podem ser identificados em nenhuma das variáveis ou índices do Sistema Compreensivo. 
Uma consideração importante sobre o DEPI é que estudos mais recentes com esta variável do Rorschach (Meyer, Viglione, Mihura, Erard, \& Erdberg, 2017; Mihura, Meyer, Dumitrascu, \& Bombel, 2013) têm deixado tácito que este índice não funciona bem, especialmente para distinguir o diagnóstico de depressão no âmbito de uma amostra clínica. O DEPI foi um dos índices de pontuação mais baixa quando se considerou uma amostra clínica. Em termos gerais, o teste de Rorschach pode proporcionar descrições da personalidade que concorrem para o diagnóstico, mas não deve ser usado como substituto de um estudo completo de diagnóstico. Além disso, muitas das variáveis componentes do índice não têm um claro fundamento no processo de resposta. Para avaliar experiências depressivas implícitas, esses estudos sugerem que seria melhor desenvolver e investigar um novo índice de depressão para o teste.

O Rorschach SC é um instrumento complexo, composto por inúmeras variáveis em que foi necessário fazer um recorte utilizando somente as variáveis relacionadas aos aspectos afetivos, as quais foram analisadas somente de forma quantitativa. Sugere-se, para estudos futuros, a integração de outros índices, tais como o de Déficit Relacional, o Índice de Desenvolvimento, o Índice de Enfraquecimento do Ego, o Índice de Perturbação do Pensamento e outras variáveis relacionadas ao Manejo e Controle do Stress para uma compreensão mais complexa do funcionamento psicológico de crianças institucionalizadas para a adoção e para diferentes formas de expressão de tristezas e angústias nessas crianças.

Como limitação deste estudo, destaca-se o seu caráter transversal, já que a avaliação foi realizada em apenas um momento, o que poderia refletir a influência de cirscuntâncias desfavoráveis atuais. Salienta-se a necessidade de estudos de follow up para averiguar a persistência e a severidade de sintomas depressivos nessas crianças. Assim, torna-se importante o acompanhamento de crianças por um tempo maior do que o realizado neste estudo.

\section{Referências}

Álvares, A. De M., \& Lobato, G. R. (2013). Um estudo exploratório da incidência de sintomas depressivos em crianças e adolescentes em acolhimento institucional. Temas em Psicologia, 21(1), 151-164. https://doi. org/10.9788/TP2013.1-11

American Psychiatric Association - APA (2014). Manual Diagnóstico e Estatístico de Transtornos Mentais - DSM 5 (5. ed.). Porto Alegre: Artimed..

Avanci, J., Assis, S., Oliveira, R., \& Pires, T. (2009). Quando a convivência com a violência aproxima a criança do comportamento depressivo. Ciência \& Saúde Coletiva, 14(2), 383-394. https://doi.org/10.1590/S1413$\underline{81232009000200008}$

Batki, A. (2017). The impact of early institutional care on emotion regulation: studying the play narratives of post-institutionalized and early adopted children. Early Child Development and Care, 188,(12), 1801-1815. https://doi.org/10.1080/03004430. 2017.1289190

Bick, J., \& Nelson, C. A. (2016). Early Adverse Experiences and the Developing Brain. Neuropsycho pharmacology, 41(1), 177-196. http://doi.org/10.1038/ npp.2015.252

Bowlby, J. (1978). Attachement et Perte. Paris: PUF.

Braithwaite, E. C., O’Connor, R. M., Degli-Esposti, M., Luke, N., \& Bowes, L. (2017). Modifiable predictors of depression following childhood maltreatment: a systematic review and meta-analysis. Translational Psychiatry, 7(7), e1162. https://doi.org/10.1038/tp.2017.140

Calcing, J., \& da Cruz Benetti, S. P. (2014). Caracterização da Saúde Mental em Crianças e Adolescentes em Acolhimento Institucional. Psico, 45(4), 559-567. https:// doi.org/10.15448/1980-8623.2014.4.13629

Calderaro, R. S. S \& Carvalho, C. V. (2005). Depressão na infância: Um estudo exploratório. Psicologia em Estudo, 10(2), 181-189. https://doi.org/10.1590/S141373722005000200004

Cohen, J. (1988). Statistical power analysis for the behavioral sciences. ( ${ }^{\mathrm{a}}$ ed). Hillsdale, New Jersey: Lawrence Erbaum. 
Copper-Royer, B. (2017). Enfant anxieux, enfant peureux. Paris: Albin Michel

Dell'Aglio, D. D., \& Hutz, C. S. (2004). Depressão e Desempenho Escolar em Crianças e Adolescentes Institucionalizados. Psicologia: Reflexão e Crítica, 17(3), 341-350. https://doi.org/10.1590/S0102$\underline{79722004000300008}$

Estatuto da Criança e do Adolescente (1990 Julho 16). Lei 8.069, de 13 de julho de 1990. D. O. pp. 13563. Atualizado com a Lei Nacional da Adoção (Lei 12.010, de 03.08.2009. D. O. 04 agosto 2009, pp. 1). https://doi. org/10.24824/978854442147.5

Exner, J. E., Jr. (2003). The Rorschach: A comprehensive system. Vol. 1: Basic foundations and principles of interpretation. Hoboken, NJ: Wiley.

Farah, F.H.Z., Cardoso, L.M. \& Villemor-Amaral, A. E. (2014). Precisão e validade do Pfister para avaliação de crianças. Avaliação Psicológica, 13(2), 187-194. https:// doi.org/10.15448/1980-8623.2016.1.19990

Goldmann, S. (2012). Developmental Epidemiology of Depressive Disorders. Child and adolescent psychiatric clinics of North America, 2l(2), 217-235. http://dx.doi. org/10.1016/j.chc.2011.12.002

Gontijo, D. T., \& Medeiros, M. (2007). Crianças e adolescentes em processo de exclusão social. Estudos, 34(1/2), 119-133. DOI INEXISTENTE

Gouveia, R. S. V. \& Gouveia, V. V. (2013). Depressão na infância e adolescência: conceituação, medida e tratamento. São Paulo: Vetor.

Kaser-Boyd, N., \& Evans, F. (2008). Rorschach assessment of psychological trauma. In: C. Gacono, \& F. Evans, The Handbook of Forensic Rorschach Assessment. (pp. 255-277). New York: Routledge. https://doi.org/10.108 $\underline{0 / 00223891.2015 .1036436}$

Luxenberg, T., \& Levin, P. (2004). The role of the Rorschach in the assessment and treatment of trauma. In: J. Wilson, \& T. Keane, Assessing psychological trauma and PTSD. (pp. 190-225). New York: Guilford.

Meyer, G. J., \& Kurtz, J. E. (2006). Guidelines Editorial - Advancing personality assessment terminology: Time to retire "objective" and "projective" as personality test descriptors. Journal of Personality Assessment, 87, 223-225. https://doi.org/10.1207/s15327752 jpa8703 01
Meyer, G. J., Viglione, D. J., Mihura, J. L., Erard, R. E., \& Erdberg, P. (2017). R-PAS - Sistema de Avaliação por Performance no Rorschach. São Paulo: Hogrefe.

Mihura, J. L., Meyer, G. J., Dumitrascu, N., \& Bombel, G. (2013). The validity of individual Rorschach variables: Systematic reviews and meta-analyses of the comprehensive system. Psychological Bulletin, 139(3), 548-605. https://doi.org/10.1037/a0029406

Milani, R. G. \& Loureiro, S. R. (2009). Crianças em risco psicossocial associado à violência doméstica: o desempenho escolar e o autoconceito como condições de proteção. Estudos de Psicologia, 14(3), 191-198. https://doi.org/10.1590/S1413-294X2009000300002

Nascimento, R. S. G. F., Resende, A.C., \& Ribeiro, R. K. S. M. (2017). Crianças e adolescentes avaliadas pelo Método de Rorschach Sistema Compreensivo. Casa do Psicólogo: São Paulo. Casa do Psicólogo. São Paulo. https://doi.org/10.11606/t.47.2008.tde-30112009-091310

Oliveira, L. de M., \& Resende, A. C. (2016). Estudo de Sintomas Depressivos em Crianças sob situação de Acolhimento Institucional. Psicologia em Pesquisa, 10(1), 55-63. https://dx.doi.org/10.24879/201600100010047

Paredes P., \& Calvete, E. (2014). Cognitive vulnerabilities as mediators between emotional abuse and depressive symptoms. Journal of Abnormal Child Psychology, 42, 743-753. https://doi.org/10.1007/s10802-013-9828-7

Peixoto, A. M. M., Silva, D. H., Pereira, D. E. I., Silva, F. G. D., Borges, H. M., Souza, I. A., Carvalho, J. T., ... Chaveiro, M. T. (2012). Da região metropolitana de Goiânia (GO): Possibilidades do olhar geográfico. Observatorium, 4(11), 138-148.

Pereira, P. C., Santos, A. B., \& Williams, L. C. A. (2009). Desempenho escolar da criança vitimizada encaminhada ao fórum judicial. Psicologia: Teoria e Pesquisa, 25(1), 19-28. https://doi.org/10.1590/S0102$\underline{37722009000100003}$

Pracana, S. M., \& Santos, S. V. (2010). Depressão em crianças e adolescentes em acolhimento institucional: Caracterização e relação com variáveis do acolhimento. Actas do VII Simpósio Nacional de Investigação em Psicologia (pp. 721-735). Braga: Universidade do Minho. https://doi.org/10.11606/d.47.2012.tde-08112012-152109 
Resende, A. C. (2016). Método de Rorschach: referências essenciais (edição revista e atualizada). Goiânia: Editora IGAP.

Resende, K. I. D. S. de, \& Teodoro, M. (2018). Avaliação da depressão infantil. In: M. Lins, M. Muniz, \& L. Cardoso, Avaliação psicológica infantil. (pp. 353-376). São Paulo: Hogrefe.

Rutter, M., Kumsta, R., Schlotz, W., \& Sonuga-Barke, E. (2012). Longitudinal studies using a "natural experiment" design: The case of adoptees from Romanian institutions. Journal of the American Academy of Child and Adolescent Psychiatry, 51(8), 762-770. https://doi. org/10.1016/j.jaac.2012.05.011

Sperry D. M., \& Widom, C, S. (2013). Child abuse and neglect, social support, and psychopathology in adulthood: a prospective investigation. Child Abuse \& Neglect, 37, 415-425. https://doi.org/10.1016/j.chiabu.2013.02.006

Spitz, R. A. (1976). De la naissance à la parole. Paris: PUF.

Vasconcelos, Q. A., Yunes, M. A. M. \& Garcia, N. M. (2009). Um estudo ecológico sobre as interações da família com o abrigo. Paidéia, 19(43), 221-229. https:// doi.org/10.1590/S0103-863X2009000200010

Wathier, J. L., Dell'Aglio, D. D.\& Bandeira, D. R. (2008). Análise fatorial do Inventário de Depressão Infantil (CDI) e amostra de jovens brasileiros. Avaliação Psicológica, 7(1), 75-84.

Weiner, I. B., \& Greene, R. L. (2008). Handbook of personality assessment. New York:Wiley.

Wulczyn, F., Brunner, K., \& Goerge, R. (2002). Multistate foster care data archive. Chicago, IL: University of Chicago Press.
Endereço para correspondência: Ana Cristina

Resende (Rua 7, № 380, apto 701, Setor Oeste, Goiânia, GO, CEP 74110090; (62) 3251-1018/Celular

(62) 99137-0535; E-mail: profa.resende@gmail.com).

Recebido em: 11/1/2018.

Aprovado em: 19/2/2019.

Publicado em: xx/xx/2019.

Ana Cristina Resende,

E-mail: profa.resende@gmail.com

Titulação Acadêmica: Doutora

ORCID: https://orcid.org/0000-0001-5730-2577

Afiliação Institucional: Pontifícia Universidade Católica de Goiás.

\section{Lorena de Melo Medonça Oliveira,}

E-mail: fran_re@yahoo.com.br

Titulação Acadêmica: Mestre

ORCID: https://orcid.org/0000-0002-7352-2833

Afiliação Institucional: Pontifícia Universidade

Católica de Goiás.

\section{Renata Franco}

E-mail: melo_oliveira@yahoo.com.br

Titulação Acadêmica: Doutora

ORCID: https://orcid.org/0000-0002-6429-1092

Afiliação Institucional: Institut Catholique de

Toulouse. 